\title{
Spatio-Temporal Dynamics of the Spatial AK Model with Trade Costs
}

\author{
Hanlei Hu${ }^{1}$, Shaoyong Lai ${ }^{2}$ \\ ${ }^{1}$ Southwest Jiaotong University, Chengdu, China \\ ${ }^{2}$ Southwestern University of Finance and Economics, Chengdu, China \\ Email: huhanlei521@sina.com
}

How to cite this paper: Hu, H.L. and Lai, S.Y. (2021) Spatio-Temporal Dynamics of the Spatial AK Model with Trade Costs. Journal of Mathematical Finance, 11, 398415.

https://doi.org/10.4236/jmf.2021.113023

Received: May 18, 2021

Accepted: July 26, 2021

Published: July 29, 2021

Copyright ( 2021 by author(s) and Scientific Research Publishing Inc. This work is licensed under the Creative Commons Attribution International License (CC BY 4.0).

http://creativecommons.org/licenses/by/4.0/

\begin{abstract}
In this paper, we introduce trade costs into the spatial AK model of economic growth and investigate the effects of trade costs on the spatio-temporal dynamics. We formulate an optimal control problem and obtain the evolution of the capital distribution along the optimal consumption trajectory via dynamic programming and semigroup theory. We also consider the convergence of the physical capital in the long-run and derive a value range for the cost coefficient that guarantee the convergence of the detrended capital across the location. Finally, we perform numerical simulations to support the analytical results and illustrate the spatiotemporal dynamics generated by the model.
\end{abstract}

\section{Keywords}

Optimal Control, Spatial AK Model, Trade Costs, Dynamic Programming

\section{Introduction}

With the development of new economic geography, capital flows and trade costs in line with the economic reality have attracted extensive attentions of researchers. Many studies have shown that trade costs play a crucial role in explaining some important economic phenomena by way of empirical analysis, see [1]-[6] and so on. In this paper, we explore the impacts of trade costs from the perspective of spatial economic growth model.

The spatial economic growth model reconcile the growth and geography economics by merging the continuous spatial dimension with the standard economic growth model, and it is an effective way to study the long-run structure of the spatial distribution of the capital stock. The motion law of physical capital in a spatial setting is described by a parabolic partial differential equation in [7], 
which lays a foundation for the following study of spatial economic growth. More recent contributions in the same stream are the study of the Benthamian case in [8], Camacho et al. propose a numerical algorithm to analyze the spatial Ramsey model [9]. Boucekkine et al. study the spatial AK model and show that the convergence of the detrended capital across space due to spatio-temporal dynamics by means of dynamic programming and generalize it to heterogeneous space, see [10] [11]. Ballestra deals with the same model in [10] by using the Pontryagin maximum principle with Michel-type transversality condition [12]. Juchem Neto and Claeyssen induced labor migration in a spatial Solow model and analyze stability of the spatially homogeneous equilibrium [13], then further consider capital transport cost in [14]. Xepapadeas and Yannacopoulos [15] develop a spatial growth model where saving rates are exogenous. Fabbri investigates the role of geography in the evolution of a spatial growth model [16]. A wider literature on spatial dynamics refers to [17]. As far as we concerned, most of the related literatures focus on how to solve the economic growth model with spatial variables and analyze the effect of spatial factor on consumption or the dynamics of capital accumulation across space. Trade costs like transportation costs, tariff and non-tariff barriers or any other mobility frictions have always been ignored. Therefore, the combination of trade cost and spatial economic growth model is our literature contribution.

Considering the importance of trade costs, we make a modification of the trade balance presented in [10] and incorporate trade costs into the spatial AK model. The main contribution of the present paper is to analyze the effects of trade costs on the spatio-temporal dynamics and the convergence of the physical capital in the long-run. Through solving an auxiliary optimal control problem, we obtain the evolution of the physical capital distribution along the optimal consumption trajectory and derived the value range of the cost coefficient that guarantees the convergence. In addition, we carry out numerical illustrations to show the dynamics of the detended capital with different trade costs.

The rest of the paper is organized as follows. Section 2 presents the spatial AK model with trade costs. Section 3 shows the analytical results on spatial-temporal dynamics. Section 4 provides complementary numerical illustrations. Section 5 concludes.

\section{The Spatial AK Model with Trade Costs}

Following [10], we assume that population is non-growing and evenly distributed on the unit circle which denoted by $\mathbb{T}$. Using polar coordinates, $\mathbb{T}$ can be described as the set of spatial parameters $\theta$ in $[0,2 \pi]$. The law of motion of capital in time and space refers to [7]. At a given point $(t, \theta) \in[0, \infty) \times \mathbb{T}$, not taking into account capital depreciation, physical capital $k(t, \theta)$ evolves according to

$$
\frac{\partial k(t, \theta)}{\partial t}=y(t, \theta)-c(t, \theta)-v(t, \theta)
$$


with capital endowments $k(0, \theta)=k_{0}(\theta)$. We consider the AK production structure, which means that the production function $y(t, \theta)$ satisfies

$$
y(t, \theta)=A k(t, \theta),
$$

where constant $A>0$ stands for technology level, and $c(t, \theta)$ and $v(t, \theta)$ represent consumption and trade balance at $(t, \theta) \in[0, \infty) \times \mathbb{T}$ respectively. Different from [10], we consider the adjustment and transportation costs when capital moving from a location to another. Then the trade balance $v(\theta, t)$ at region $\Theta_{i} \in \mathbb{T}$ could be described as

$$
\int_{\Theta_{i}} v(t, \theta) \mathrm{d} \theta=-\left[\frac{\partial k\left(t, \theta_{i}+\Delta \theta_{i}\right)}{\partial \theta}-\frac{\partial k\left(t, \theta_{i}\right)}{\partial \theta}\right]-b k(t, \theta),
$$

where $b(b>0)$ is the barrier measure of the capital $k(t, \theta)$.

Using the fundamental theorem of calculus yields

$$
\frac{\partial k\left(t, \theta_{i}+\Delta \theta_{i}\right)}{\partial \theta}-\frac{\partial k\left(t, \theta_{i}\right)}{\partial \theta}=\int_{\theta_{i}}^{\theta_{i}+\Delta \theta_{i}} \frac{\partial}{\partial \theta} \frac{\partial k(t, \theta)}{\partial \theta} \mathrm{d} \theta=\int_{\Theta_{i}} \frac{\partial^{2} k(t, \theta)}{\partial \theta^{2}} \mathrm{~d} \theta .
$$

Thus the trade balance (3) can be written in the form

$$
\int_{\Theta_{i}} v(t, \theta) \mathrm{d} \theta=-\int_{\Theta_{i}} \frac{\partial k^{2}(t, \theta)}{\partial \theta^{2}} \mathrm{~d} \theta-b k(t, \theta),
$$

which derives

$$
v(t, \theta)=-\frac{\partial k^{2}(t, \theta)}{\partial \theta^{2}}-b \frac{\partial k(t, \theta)}{\partial \theta} .
$$

Substituting (2) and (6) into Equation (1), the dynamic of the physical capital accumulation is represented by a general parabolic partial differential equation

$$
\frac{\partial k(t, \theta)}{\partial t}=A(t) k(t, \theta)-c(t, \theta)+\frac{\partial k^{2}(t, \theta)}{\partial \theta^{2}}+b \frac{\partial k(t, \theta)}{\partial \theta}
$$

with nonnegative initial capital distribution $k(0, \theta)=k_{0}(\theta)$. The term $\frac{\partial^{2} k(t, \theta)}{\partial \theta^{2}}+b \frac{\partial k(t, \theta)}{\partial \theta}$ in Equation (7) captures capital mobility across space and $b \frac{\partial k(t, \theta)}{\partial \theta}$ represents trade costs like adjustment costs, institutional barriers or other mobility frictions in the trade balance. This term make a difference with [10] and it is the key to discuss the spatial dynamics of the growth model with trade costs.

To complete the model, we impose a nonhomogeneous Dirichlet boundary condition

$$
k(t, 0)=k(t, 2 \pi)
$$

as in [10]. It is clear that the value of the capital stock $k(t, \theta)$ must be nonnegative everywhere and in any moment of time. In addition, the initial distribution of capital $k_{0}(\theta)$ is assumed to be known, bounded and continuous, $c(\theta, t)$ is smooth, bounded and concave. Putting all these pieces together, our model 
becomes the following partial differential system

$$
\left\{\begin{array}{l}
\frac{\partial k(t, \theta)}{\partial t}=\frac{\partial^{2} k(t, \theta)}{\partial \theta^{2}}+b \frac{\partial k(t, \theta)}{\partial \theta}+A k(t, \theta)-c(t, \theta), \\
k(0, \theta)=k_{0}(\theta), \theta \in \mathbb{T}, \\
k(t, 0)=k(t, 2 \pi), t \in[0,+\infty) .
\end{array}\right.
$$

Comparing system (9) with the model analyzed in [10], the difference is the term $b k_{\theta}(t, \theta)$, which models trade costs. The present work aim to discuss how this term affects the spatial dynamics of the economic model. We consider a centralized economy in which a central planner finds a flow of optimal distributions of consumption, subject to a spatial-temporal capital accumulation budget constraint. The problem of optimal growth in dynamic spatial economy is that the social planner maximizes a sum of utilities of all regions,

$$
J\left(k_{0}, c(\cdot, \cdot)\right):=\int_{0}^{+\infty} \mathrm{e}^{-\rho t} \int_{0}^{2 \pi} U(c(t, \theta)) \mathrm{d} \theta \mathrm{d} t,
$$

where $\rho>0$ is the rate of time preference and $U(c(t, \theta))=\frac{c(t, \theta)^{1-\sigma}}{1-\sigma}$ is the instantaneous utility function, subject to an initial and boundary problem of the parabolic partial differential system (9). Our main work is to find the optimal consumption strategy and discuss the spatial impact of trade costs on the dynamics of capital growth.

We claim that the functions $k(\cdot, \cdot), c(\cdot, \cdot)$ involved in this paper are regular enough. For all $t \in[0,+\infty), k(t, \cdot), c(t, \cdot)$ of the space variable can be considered as elements of the Hilbert space $L^{2}(\mathbb{T})$ whose elements are functions. The scalar product of $f$ and $g$ in $L^{2}(\mathbb{T})$ is defined as

$$
\langle f, g\rangle:=\int_{0}^{2 \pi} f(\theta) g(\theta) \mathrm{d} \theta
$$

and $\int_{0}^{2 \pi}|f(\theta)|^{2} \mathrm{~d} \theta<+\infty$. The norm in $L^{2}(\mathbb{T})$ is given by

$$
|f|_{L^{2}(\mathbb{T})}:=\langle f, f\rangle^{\frac{1}{2}}=\left[\int_{0}^{2 \pi} f^{2}(\theta) \mathrm{d} \theta\right]^{\frac{1}{2}} .
$$

This allows us to apply dynamic programming approach and solve Hamilton-Jacobi-Bellman equation in $L^{2}(\mathbb{T})$. Furthermore, we could find the optimal control in feedback form.

\section{Spatio-Temporal Dynamics: Analytical Results}

Before solving the optimization problem (10), we consider an auxiliary problem in the following Lemma.

Lemma 1 (Auxiliary Problem) Let $z(t, \theta)$ be a positive function defined in $L^{2}(\mathbb{T})$, and the objective function

$$
J\left(z_{0}\right)=\sup _{\hat{c}(\cdot, \cdot)} \int_{0}^{+\infty} \mathrm{e}^{-\rho t} \int_{0}^{2 \pi} \frac{[u(\theta) \hat{c}(t, \theta)]^{1-\sigma}}{1-\sigma} \mathrm{d} \theta \mathrm{d} t,
$$

subject to 


$$
\left\{\begin{array}{l}
\frac{\partial z(t, \theta)}{\partial t}=\frac{\partial^{2} z(t, \theta)}{\partial \theta^{2}}+\left(A-\frac{b^{2}}{4}\right) z(t, \theta)-\hat{c}(t, \theta), \\
z(0, \theta)=z_{0}(\theta), \theta \in \mathbb{T}, \\
z(t, 0)=\mathrm{e}^{-b \pi} z(t, 2 \pi), t \in[0,+\infty) .
\end{array}\right.
$$

There exists $u(\theta)$ make system (14) equivalent to system (9) where $\hat{c}(t, \theta)=u^{-1}(\theta) c(t, \theta)$.

Proof Suppose $u(\theta)=\exp \left\{-\frac{b \theta}{2}\right\}$. For all $(t, \theta) \in[0,+\infty) \times \mathbb{T}$, let

$$
k(t, \theta)=z(t, \theta) u(\theta),
$$

we have

$$
\begin{gathered}
\frac{\partial k(t, \theta)}{\partial t}=\frac{\partial z(t, \theta)}{\partial t} u(\theta), \\
\frac{\partial k(t, \theta)}{\partial \theta}=\frac{\partial z(t, \theta)}{\partial \theta} u(\theta)-\frac{b}{2} z(t, \theta) u(\theta), \\
\frac{\partial^{2} k(t, \theta)}{\partial \theta^{2}}=\frac{\partial^{2} z(t, \theta)}{\partial \theta^{2}} u(\theta)-b \frac{\partial z(t, \theta)}{\partial \theta} u(\theta)+\frac{b^{2}}{4} z(t, \theta) u(\theta) .
\end{gathered}
$$

Then

$$
\frac{\partial^{2} k(t, \theta)}{\partial \theta^{2}}+b \frac{\partial k(t, \theta)}{\partial \theta}+A k(t, \theta)=\left[\frac{\partial z^{2}(t, \theta)}{\partial \theta^{2}}+\left(A-\frac{b^{2}}{4}\right) z(t, \theta)\right] u(\theta) .
$$

Plugging Equations (15-19) into the first equation of system (9), and divide both sides by $u(\theta)$, we get

$$
\frac{\partial z(t, \theta)}{\partial t}=\frac{\partial^{2} z(t, \theta)}{\partial \theta^{2}}+\left(A-\frac{b^{2}}{4}\right) z(t, \theta)-u^{-1}(\theta) c(t, \theta),
$$

denote $u^{-1}(\theta) c(t, \theta)=\hat{c}(t, \theta)$. Furthermore, we have

$$
\begin{gathered}
z(0, \theta)=u^{-1}(\theta) k(0, \theta)=u^{-1}(\theta) k_{0}(\theta), \\
z(t, 0)=u^{-1}(0) k(t, 0)=u^{-1}(0) k(t, 2 \pi)=u^{-1}(0) u(2 \pi) z(t, 2 \pi) .
\end{gathered}
$$

Thus, the initial and boundary conditions

$$
z_{0}(\theta)=u^{-1}(\theta) k_{0}(\theta), z(t, 0)=\mathrm{e}^{-b \pi} z(t, 2 \pi)
$$

are verified. Then system (14) is proved to be equivalent to system (9).

Note that if we choose $b=0$, there will be no trade costs in the economy. In this case, the auxiliary problem is exactly the system considered in [10]. The next step is to analyze the auxiliary problem, from which we can solve the original optimization problem.

We define the value function of problem (13) starting from $z_{0}$ as

$$
V\left(z_{0}\right):=\sup _{\hat{c}(\cdot, \cdot)} J\left(z_{0}, \hat{c}(\cdot, \cdot)\right) .
$$

Define the operator $G$ in $L^{2}(\mathbb{T})$ in the form of 


$$
G(f)=\frac{\partial^{2} f}{\partial \theta^{2}},
$$

denote the domain of $G$ as $D(G)$, which is a subset of $L^{2}(\mathbb{T})$. Given an initial distribution of capital $k_{0}(\theta) \in L^{2}(\mathbb{T})$, which is a function of the space variable $\theta$. From Lemma 1 , we have $z_{0}(\theta)=\mathrm{e}^{\frac{b \theta}{2}} k_{0}(\theta)$, thus $z_{0}(\theta) \in L^{2}(\mathbb{T})$. Referring to [10], we know that the expression $\mathrm{e}^{t G} z_{0}(\theta) \in L^{2}(\mathbb{T})$ for all $t \geq 0$ and $\mathrm{e}^{t G} z_{0}(\theta)$ is the unique solution of

$$
\left\{\begin{array}{l}
\frac{\partial z(t, \theta)}{\partial t}=\frac{\partial^{2} z(t, \theta)}{\partial \theta^{2}}=G(z(t, \theta)), \\
z(0, \theta)=z_{0}(\theta), \forall \theta \in \mathbb{T} \\
z(t, 0)=\mathrm{e}^{-b \pi} z(t, 2 \pi), \forall t \geq 0
\end{array}\right.
$$

at time $t$. That is, for a fixed $t$ the expression $\mathrm{e}^{t G} z_{0}(\theta)$ is a function of the space variable $\theta$ and $\mathrm{e}^{t G} z_{0}(\theta)=z(t, \theta)$, where $z(\cdot, \cdot)$ satisfies system (26). Furthermore, for $z_{0} \in D(G)$,

$$
\frac{\partial z(t, \theta)}{\partial t}:=\lim _{h \rightarrow 0} \frac{z(t+h, \theta)-z(t, \theta)}{t}=\lim _{h \rightarrow 0} \frac{\mathrm{e}^{(t+h) G} z_{0}(\theta)-\mathrm{e}^{t G} z_{0}(\theta)}{t} .
$$

Since $G z(t, \theta)=G e^{t G} z_{0}(\theta)$, we have

$$
\lim _{h \rightarrow 0} \frac{\mathrm{e}^{(t+h) G} z_{0}(\theta)-\mathrm{e}^{t G} z_{0}(\theta)}{t}=G \mathrm{e}^{t G} z_{0}(\theta), \forall t \geq 0 .
$$

i.e.

$$
\frac{\mathrm{de}^{t G} z_{0}(\theta)}{\mathrm{d} t}=G \mathrm{e}^{t G} z_{0}(\theta) .
$$

Hence, the expression $\mathrm{e}^{t G}$ is said to be generated by $G$ and satisfies "semigroup property": for all $z_{0}(\theta) \in L^{2}(\mathbb{T})$ and $s, t \geq 0$, $\mathrm{e}^{(t+s) G} z_{0}=\mathrm{e}^{s G}\left(\mathrm{e}^{t G} z_{0}\right)$.

Combining with the definition of the operator $G$, the state Equation in (14) can be rewritten as an evolution equation in $L^{2}(\mathbb{T})$, namely,

$$
\left\{\begin{array}{l}
\dot{z}(t)=G z(t)+\left(A-\frac{b^{2}}{4}\right) z(t)-\hat{c}(t), \\
z(0)=z_{0},
\end{array}\right.
$$

where $\dot{z}(\cdot)$ is time derivative. Note that $z(t), \hat{c}(t) \in L^{2}(\mathbb{T}), \forall t \geq 0$, that means $z(t)$ and $\hat{c}(t)$ are functions of space, i.e. $z(t)(\theta) \equiv z(t, \theta)$, $\hat{c}(t)(\theta) \equiv \hat{c}(t, \theta)$. The set of admissible controls is $\mathcal{U}_{z_{0}}:=\left\{\hat{c} \in L_{\text {loc }}^{2}\left(\mathbb{R}^{+} ; L^{2}(\mathbb{T})\right): \hat{c}(t)(\theta), z(t)(\theta) \geq 0, \forall(t, \theta) \in \mathbb{R}^{+} \times \mathbb{T}\right\}$. Accordingly, the value function rewrite as $V\left(z_{0}\right):=\sup _{\hat{c}(\cdot) \in \mathcal{u}_{z_{0}}} J\left(z_{0}, \hat{c}(\cdot)\right)$.

We define function $\mathbb{1}: \mathbb{T} \rightarrow \mathbb{R}$ as the constant (in the space variable) equal to 1 , i.e. $\mathbb{1}(\theta) \equiv 1$. Then the optimization problem (13) transformed into

$$
J\left(z_{0}, \hat{c}(\cdot, \cdot)\right)=\sup _{\hat{c}(\cdot)} \int_{0}^{+\infty} \mathrm{e}^{-\rho t}\langle\mathbb{1}, U(u(\theta) \hat{c}(t))\rangle \mathrm{d} t,
$$


where $U(f): \mathbb{T} \rightarrow \mathbb{R}$ is the function $U(f)(\theta)=\frac{f(\theta)^{1-\sigma}}{1-\sigma}$ for a given $f \in L^{2}(\mathbb{T})$, subject to system (30).

Applying the dynamic programming approach, the corresponding Hamilton-Jacobi-Bellman(HJB) equation of problem (31) is written as

$$
\begin{aligned}
\rho v(z)= & \langle z, G \nabla v(z)\rangle+\left(A-\frac{b^{2}}{4}\right)\langle z, \nabla v(z)\rangle \\
& +\sup _{\hat{c} \in L^{2}\left(\mathbb{T}, \mathbb{R}^{+}\right)}\left\{-\langle\hat{c}, \nabla v(z)\rangle+\left\langle\mathbb{1}, U\left(\mathrm{e}^{-\frac{b \theta}{2}} \hat{c}\right)\right\rangle\right\},
\end{aligned}
$$

where $\nabla$ represents Gàteaux derivative, and we can write $\langle z, G \nabla v(z)\rangle$ instead of $\langle G z, \nabla v(z)\rangle$, because $G$ is self-adjoint. Note that $v: L^{2}(\mathbb{T}) \rightarrow \mathbb{R}$, i.e. for every $z \in L^{2}(\mathbb{T}), v(z)$ is a real number, being $z$ in itself a function from $\mathbb{T}$ to $\mathbb{R}$. According to the optimal control theory, we need to find the solution $v(z)$ of Equation (32) and prove it equals to the value function $V\left(z_{0}\right)$.

Lemma 2 Given a positive function $z_{0} \in L^{2}(\mathbb{T})$, suppose that

$$
2 \sqrt{A-\frac{\rho}{1-\sigma}}<b<2 \sqrt{A} \text {, }
$$

and let

$$
\eta:=\frac{\rho-\left(A-\frac{b^{2}}{4}\right)(1-\sigma)}{2 \pi \sigma} .
$$

Provided that the trajectory $z^{*}(t, \theta)$, driven by the feedback control (constant in $\theta$ )

$$
\hat{c}^{*}(t, \theta)=\eta \int_{0}^{2 \pi} z^{*}(t, \varphi) \mathrm{d} \varphi,
$$

remains positive, $\hat{c}^{*}(t, \theta)$ is the unique optimal control of the problem (14). The value function of the problem (14) is written explicitly in the form

$$
V\left(z_{0}\right)=\alpha\left(\int_{0}^{2 \pi} z_{0}(\theta) \mathrm{d} \theta\right)^{1-\sigma}
$$

where

$$
\alpha=\left[\frac{\rho-\left(A-\frac{b^{2}}{4}\right)(1-\sigma)}{2 \pi \sigma}\right]^{-\sigma} \cdot \frac{B}{2 \pi(1-\sigma)} .
$$

Proof Firstly, we look for a solution of Equation (32) in the form of

$$
v(z)=\alpha\langle z, \mathbb{1}\rangle^{1-\sigma},
$$

where $\alpha$ is a positive coefficient to be determined, so that

$$
\nabla v(z)=\alpha(1-\sigma)\langle z, \mathbb{1}\rangle^{-\sigma} \mathbb{1}
$$

Substituting $v(z), \nabla v(z)$ into Equation (32), we obtain 


$$
\begin{aligned}
\rho \alpha\langle z, \mathbb{1}\rangle^{1-\sigma}= & \alpha(1-\sigma)\langle z, \mathbb{1}\rangle^{-\sigma}\langle z, G \mathbb{1}\rangle+\left(A-\frac{b^{2}}{4}\right) \alpha(1-\sigma)\langle z, \mathbb{1}\rangle^{-\sigma}\langle z, \mathbb{1}\rangle \\
& +\sup _{\hat{c} \in \mathcal{U}_{z 0}}\left\{-\alpha(1-\sigma)\langle z, \mathbb{1}\rangle^{-\sigma}\langle\hat{c}, \mathbb{1}\rangle+\left\langle\mathbb{1}, U\left(\mathrm{e}^{-\frac{b \theta}{2}} \hat{c}\right)\right\rangle\right\} .
\end{aligned}
$$

Denote

$$
H(\hat{c})=\sup _{\hat{c} \in \mathcal{U}_{z_{0}}}\left\{-\alpha(1-\sigma)\langle z, \mathbb{1}\rangle^{-\sigma}\langle\hat{c}, \mathbb{1}\rangle+\left\langle\mathbb{1}, U\left(\mathrm{e}^{-\frac{b \theta}{2}} \hat{c}\right)\right\rangle\right\} .
$$

By the definition of scalar product (11), we get

$$
\begin{aligned}
\langle\hat{c}, \mathbb{1}\rangle & =\int_{0}^{2 \pi} \hat{c}(t) \mathrm{d} \theta=2 \pi \hat{c}(t), \\
\left\langle U\left(\mathrm{e}^{-\frac{b \theta}{2}} \hat{c}\right), \mathbb{1}\right\rangle & =\int_{0}^{2 \pi} \frac{\left[\mathrm{e}^{-\frac{b \theta}{2}} \hat{c}(t)\right]^{1-\sigma}}{1-\sigma} \mathrm{d} \theta \\
& =\frac{\hat{c}(t)^{1-\sigma}}{1-\sigma}\left[-\frac{2}{b(1-\sigma)}\left(\mathrm{e}^{-b(1-\sigma) \pi}-1\right)\right] .
\end{aligned}
$$

Take the first-order condition of Equation (41) with respect to $\hat{c}(t)$, we obtain

$$
-2 \pi \alpha(1-\sigma)\langle z, \mathbb{1}\rangle^{-\sigma}+\left[-\frac{2}{b(1-\sigma)}\left(\mathrm{e}^{-b(1-\sigma) \pi}-1\right)\right] \hat{c}(t)^{-\sigma}=0,
$$

from which we have

$$
\hat{c}(t)=\left[\frac{2 \pi}{B} \alpha(1-\sigma)\right]^{-\frac{1}{\sigma}}\langle z, \mathbb{1}\rangle \mathbb{1},
$$

where $B=-\frac{2}{b(1-\sigma)}\left(\mathrm{e}^{-b(1-\sigma) \pi}-1\right)$. So $H(\hat{c})$ attains the supremum when $\hat{c}(t)$ satisfies Equation (45).

Substituting Equation (45) into Equation (40), observing that $G \mathbb{1}=0$, we have

$$
\begin{aligned}
\rho \alpha\langle z, \mathbb{1}\rangle^{1-\sigma}= & \left(A-\frac{b^{2}}{4}\right) \alpha(1-\sigma)\langle z, \mathbb{1}\rangle^{1-\sigma} \\
& -2 \pi \alpha(1-\sigma)\langle z, \mathbb{1}\rangle^{-\sigma}\left[\frac{2 \pi}{B} \alpha(1-\sigma)\right]^{-\frac{1}{\sigma}}\langle z, \mathbb{1}\rangle \\
& +\frac{B}{1-\sigma}\left[\frac{2 \pi}{B} \alpha(1-\sigma)\right]^{1-\frac{1}{\sigma}}\langle z, \mathbb{1}\rangle^{1-\sigma} .
\end{aligned}
$$

Simplified Equation (46), we obtain

$$
\rho=\left(A-\frac{b^{2}}{4}\right)(1-\sigma)+2 \pi \sigma\left[\frac{2 \pi}{B} \alpha(1-\sigma)\right]^{-\frac{1}{\sigma}},
$$

from which we know that there exists a solution of Equation (32) when 


$$
\alpha=\frac{B}{2 \pi(1-\sigma)}\left[\frac{\rho-\left(A-\frac{b^{2}}{4}\right)(1-\sigma)}{2 \pi \sigma}\right]^{-\sigma} .
$$

Secondly, the feedback control provided by the solution (38) is $\phi(z): L^{2}(\mathbb{T}) \rightarrow L^{2}(\mathbb{T})$, we have

$$
\begin{aligned}
\phi(z) & :=\arg \max _{\hat{c} \in L^{2}(\mathbb{T})}\left\{\alpha(1-\sigma)\langle z, \mathbb{1}\rangle^{-\sigma}\langle\hat{c}, \mathbb{1}\rangle+\left\langle\mathbb{1}, U\left(\mathrm{e}^{-\frac{b \theta}{2}} \hat{c}\right)\right\rangle\right\} \\
& =\left[\frac{2 \pi}{B} \alpha(1-\sigma)\right]^{-\frac{1}{\sigma}}\langle z, \mathbb{1}\rangle \mathbb{1} \\
& =\eta\langle z, \mathbb{1}\rangle \mathbb{1},
\end{aligned}
$$

where $\eta=\left[\frac{2 \pi}{B} \alpha(1-\sigma)\right]^{-\frac{1}{\sigma}}=\frac{\rho-\left(A-\frac{b^{2}}{4}\right)(1-\sigma)}{2 \pi \sigma}$.

The related trajectory $z(t)$ satisfies the following integral equation

$$
z(t)=\mathrm{e}^{t\left(A-\frac{b^{2}}{4}+G\right)} z_{0}-\int_{0}^{t} \mathrm{e}^{(t-s)\left(A-\frac{b^{2}}{4}+G\right)} \eta\langle z(s), \mathbb{1}\rangle \mathbb{1} \mathrm{d} s .
$$

Though we can not get the exact expression, there exists a unique solution $z^{*}(t)$ of Equation (50) (see for [18]). The control that we want to prove to be admissible is $\hat{c}^{*}(t):=\phi\left(z^{*}(t)\right), \forall t \geq 0$. Since hypothesis $z^{*}(t)(\theta)$ remains positive, then $\hat{c}^{*}(t)(\theta)$ remains positive too and then it is admissible.

Thirdly, $\hat{c}^{*}(\cdot)$ is an optimal control if for any other admissible control $\tilde{c}(\cdot)$, we have $J\left(z_{0}, \hat{c}^{*}(\cdot)\right) \geq J\left(z_{0}, \tilde{c}(\cdot)\right)$. For an admissible control $\hat{c}(\cdot)$, the related trajectory $z(\cdot)$ satisfies Equation (30). $\bar{z}(t)$ is the solution of

$$
\dot{\bar{Z}}(t)=G \bar{Z}(t)+\left(A-\frac{b^{2}}{4}\right) \bar{z}(t), \bar{z}(0)=z_{0} .
$$

By the comparison theorem, for any $(t, \theta) \in \Omega$, we have $z(\cdot) \leq \bar{z}(t)$. In particular, $\langle\bar{z}(t), \mathbb{1}\rangle \geq\langle z(t), \mathbb{1}\rangle, \forall t \geq 0 . \bar{z}(t)$ can be expressed as $\bar{z}(t)=\mathrm{e}^{t\left(A-\frac{b^{2}}{4}\right)} \mathrm{e}^{t G} z_{0}$, so that $\langle\bar{z}(t), \mathbb{1}\rangle=\mathrm{e}^{t\left(A-\frac{b^{2}}{4}\right)}\left\langle\bar{z}_{0}, \mathbb{1}\right\rangle$. This means that for every choice of $\hat{c}(\cdot)$, we have

$$
\begin{aligned}
\left|\mathrm{e}^{-\rho t} v(z(t))\right| & =\mathrm{e}^{-\rho t} \alpha\langle z(t), \mathbb{1}\rangle^{1-\sigma} \\
& \leq \mathrm{e}^{-\rho t} \alpha\langle\bar{z}(t), \mathbb{1}\rangle^{1-\sigma} \\
& =\mathrm{e}^{-\rho t} \alpha\left[\mathrm{e}^{t\left(A-\frac{b^{2}}{4}\right)}\right]^{1-\sigma}\left\langle\bar{z}_{0}, \mathbb{1}\right\rangle^{1-\sigma} .
\end{aligned}
$$

When $2 \sqrt{A-\frac{\rho}{1-\sigma}}<b<2 \sqrt{A}$, 


$$
\left|\mathrm{e}^{-\rho t} v(z(t))\right| \stackrel{t \rightarrow \infty}{\longrightarrow} 0 .
$$

Let us call $\tilde{z}(\cdot)$ the trajectory relating to the admissible control $\tilde{c}(\cdot)$ and denote $w(t, z):=\mathrm{e}^{-\rho t} v(z)$. We have

$$
\begin{aligned}
& v\left(z_{0}\right)-w(T, \tilde{z}(T)) \\
& =w(t, \tilde{z}(0))-w(T, \tilde{z}(T))=-\int_{0}^{T} \frac{\mathrm{d}}{\mathrm{d} t} w(t, \tilde{z}(t)) \mathrm{d} t \\
& =\int_{0}^{T} \mathrm{e}^{-\rho t}\left[\rho v(\tilde{z}(t))-\left\langle G \tilde{z}(t)+\left(A-\frac{b^{2}}{4}\right) \tilde{z}(t)-\tilde{c}(t), \nabla v(\tilde{z}(t))\right\rangle\right] \mathrm{d} t .
\end{aligned}
$$

Passing to the limit in Equation (54) as $t \rightarrow \infty$ and using (53), we have

$$
v\left(z_{0}\right)=\int_{0}^{+\infty} \mathrm{e}^{-\rho t}\left[\rho v(\tilde{z}(t))-\left\langle\left(A-\frac{b^{2}}{4}\right) \tilde{z}(t)-\tilde{c}(t), \nabla v(\tilde{z}(t))\right\rangle-\langle\tilde{z}(t), G \nabla v(\tilde{z}(t))\rangle\right] \mathrm{d} t .
$$

Recalling HJB Equation (32), we have

$$
\begin{aligned}
& v\left(z_{0}\right)-J\left(z_{0}, \tilde{c}(\cdot)\right) \\
& =\int_{0}^{+\infty} \mathrm{e}^{-\rho t}\left[\rho v(\tilde{z}(t))-\left\langle\left(A-\frac{b^{2}}{4}\right) \tilde{z}(t), \nabla v(\tilde{z}(t))\right\rangle-\langle\tilde{z}(t), G \nabla v(\tilde{z}(t))\rangle\right. \\
& +\langle\tilde{c}(t), \nabla v(\tilde{z}(t))\rangle-\left\langle\mathbb{1}, U\left(\mathrm{e}^{-\frac{b \theta}{2}} \tilde{c}(t)\right)\right\rangle \mathrm{d} t \\
& =\int_{0}^{+\infty} \mathrm{e}^{-\rho t}\left[\sup _{\hat{c} \in L^{2}\left(\mathbb{T}, \mathbb{R}^{+}\right)}\left\{\langle\hat{c}(t), \nabla v(\tilde{z}(t))\rangle-\left\langle\mathbb{1}, U\left(\mathrm{e}^{-\frac{b \theta}{2}} \hat{c}(t)\right)\right\rangle\right\}\right. \\
& +\langle\tilde{c}(t), \nabla v(\tilde{z}(t))\rangle-\left\langle\mathbb{1}, U\left(\mathrm{e}^{-\frac{b \theta}{2}} \tilde{c}(t)\right)\right\rangle \mathrm{d} t \geq 0 .
\end{aligned}
$$

Inequality (56) shows that $v\left(z_{0}\right) \geq J\left(z_{0}, \tilde{c}(\cdot)\right)$. Since $\hat{c}^{*}(\cdot)$ is defined by feedback control (49), we have $v\left(z_{0}\right)-J\left(z_{0}, \hat{c}^{*}(\cdot)\right)=0$. Hence, for all admissible $\tilde{c}(\cdot)$, by $v\left(z_{0}\right)-J\left(z_{0}, \tilde{c}(\cdot)\right) \geq 0=v\left(z_{0}\right)-J\left(z_{0}, \hat{c}^{*}(\cdot)\right)$, we get $J\left(z_{0}, \tilde{c}(\cdot)\right) \leq J\left(z_{0}, \hat{c}^{*}(\cdot)\right)$, which implies that $\hat{c}^{*}(\cdot)$ is optimal. In particular, since $v\left(z_{0}\right)-J\left(z_{0}, \hat{c}^{*}(\cdot)\right)=0$ and $\hat{c}^{*}$ is an optimal control, $v\left(z_{0}\right)$ is the value function at $z_{0}$.

Theorem 3 Under the same assumptions of Lemma 1 and 2, the optimal trajectories $c^{*}(t, \theta)$ could be expressed as

$$
c^{*}(t, \theta)=\eta \mathrm{e}^{-\frac{b \theta}{2}+\beta t} Z(0)
$$

where

$$
\beta=\frac{\left(A-\frac{b^{2}}{4}\right)-\rho}{\sigma}
$$

and $Z(0):=\int_{0}^{2 \pi} z_{0}(\theta) \mathrm{d} \theta$. Along the optimal trajectories $c^{*}(t, \theta)$, the aggregate capital $K(t)$ is 


$$
K(t)=\mathrm{e}^{\left(A-\frac{b^{2}}{4}\right) t} K(0),
$$

where $K(0):=\int_{0}^{2 \pi} k_{0}(\theta) \mathrm{d} \theta$ is the initial level of aggregate capital.

Proof At the time $t$, the aggregate capital is equal to the sum of all the capital amounts distributed in $\mathbb{T}$,

$$
K(t):=\langle k(t, \theta), \mathbb{1}\rangle=\int_{0}^{2 \pi} k(t, \theta) \mathrm{d} \theta .
$$

From Lemma 1, There is a corresponding relationship between $k(t, \theta)$ and $z(t, \theta)$. The aggregate capital $K^{*}(t)$ along the optimal trajectory $c^{*}(t)$ is defined by

$$
\begin{aligned}
K^{*}(t) & =\left\langle k^{*}(t, \theta), \mathbb{1}\right\rangle \\
& =\left\langle z^{*}(t, \theta) u(\theta), \mathbb{1}\right\rangle \\
& =\int_{0}^{2 \pi} z^{*}(t, \theta) \mathrm{e}^{-\frac{b \theta}{2}} \mathrm{~d} \theta \\
& =-\frac{2}{b}\left[\left.z^{*}(t, \theta) \mathrm{e}^{-\frac{b \theta}{2}}\right|_{0} ^{2 \pi}-\int_{0}^{2 \pi} \mathrm{e}^{-\frac{b \theta}{2}} \mathrm{~d} z^{*}(t, \theta)\right] .
\end{aligned}
$$

Since $z^{*}(t, \theta)$ solves the mild Equation (50), we have

$$
\begin{aligned}
z^{*}(t) & =\mathrm{e}^{t\left(A-\frac{b^{2}}{4}+G\right)} z_{0}-\int_{0}^{t} \mathrm{e}^{(t-s)\left(A-\frac{b^{2}}{4}+G\right)} \hat{C}^{*}(s) \mathrm{d} s \\
& =\mathrm{e}^{t\left(A-\frac{b^{2}}{4}+G\right)} z_{0}-\int_{0}^{t} \mathrm{e}^{(t-s)\left(A-\frac{b^{2}}{4}+G\right)} \eta\left\langle z^{*}(s), \mathbb{1}\right\rangle \mathbb{1} \mathrm{d} s .
\end{aligned}
$$

Note that $\mathrm{e}^{G t} \mathbb{1}=\mathbb{1},\langle\mathbb{1}, \mathbb{1}\rangle=\int_{0}^{2 \pi} 1 \mathrm{~d} \theta=2 \pi$. Taking the scalar product of both sides of Equation (62) with $\mathbb{1} \in L^{2}(\mathbb{T})$, we obtain

$$
\begin{aligned}
Z^{*}(t) & =\left\langle z^{*}(t), \mathbb{1}\right\rangle \\
& =\mathrm{e}^{t\left(A-\frac{b^{2}}{4}\right)}\left\langle z_{0}, \mathrm{e}^{t G} \mathbb{1}\right\rangle-\int_{0}^{t} \mathrm{e}^{(t-s)\left(A-\frac{b^{2}}{4}\right)}\left\langle\eta\left\langle z^{*}(s), \mathbb{1}\right\rangle \mathbb{1}, \mathrm{e}^{(t-s) G} \mathbb{1}\right\rangle \mathrm{d} s \\
& =\mathrm{e}^{t\left(A-\frac{b^{2}}{4}\right)} Z(0)-\int_{0}^{t} \mathrm{e}^{(t-s)\left(A-\frac{b^{2}}{4}\right)} Z^{*}(s)\left[2 \pi \frac{\rho-\left(A-\frac{b^{2}}{4}\right)(1-\sigma)}{2 \pi \sigma}\right] \mathrm{d} s \\
& =\mathrm{e}^{t\left(A-\frac{b^{2}}{4}\right)} Z(0)-\int_{0}^{t} \mathrm{e}^{(t-s)\left(A-\frac{b^{2}}{4}\right)} \frac{\rho-\left(A-\frac{b^{2}}{4}\right)(1-\sigma)}{\sigma} Z^{*}(s) \mathrm{d} s .
\end{aligned}
$$

Equation (63) is a standard one-dimensional ordinary differential equation of $Z^{*}(t)$ in integral form. One can prove by inspection that Equation (63) has a unique solution

$$
Z^{*}(t)=Z(0) \mathrm{e}^{\beta t}
$$

with $\beta=\frac{\left(A-\frac{b^{2}}{4}\right)-\rho}{\sigma}$. Therefore, $\left\langle z^{*}(s), \mathbb{1}\right\rangle=Z(s)=Z(0) \mathrm{e}^{\beta s}$, which im- 
plies that $\int_{0}^{t} \mathrm{e}^{(t-s)\left(A-\frac{b^{2}}{4}+G\right)} \eta\left\langle z^{*}(s), \mathbb{1}\right\rangle \mathbb{1} \mathrm{d} s$ is constant with respect to $\theta$. By Equation (15), we obtain

$$
\begin{aligned}
\mathrm{d} z^{*}(t, \theta) & =\mathrm{e}^{t\left(A-\frac{b^{2}}{4}+G\right)} \mathrm{d} z(0, \theta) \\
& =\mathrm{e}^{t\left(A-\frac{b^{2}}{4}+G\right)} \mathrm{d}\left[k(0, \theta) u^{-1}(\theta)\right] \\
& =\mathrm{e}^{t\left(A-\frac{b^{2}}{4}+G\right)}\left[k(0, \theta) \mathrm{de}^{\frac{b \theta}{2}}+\mathrm{e}^{\frac{b \theta}{2}} \mathrm{~d} k(0, \theta)\right] .
\end{aligned}
$$

Recall that $z(t, 0)=\mathrm{e}^{-b \pi} z(t, 2 \pi),\left.\quad z(t, \theta) \mathrm{e}^{-\frac{b \theta}{2}}\right|_{0} ^{2 \pi}=0$, we have

$$
\begin{aligned}
K^{*}(t) & =\frac{2}{b} \int_{0}^{2 \pi} \mathrm{e}^{-\frac{b \theta}{2}} \mathrm{~d} z(t, \theta) \\
& =\frac{2}{b} \int_{0}^{2 \pi} \mathrm{e}^{-\frac{b \theta}{2}} \mathrm{e}^{t\left(A-\frac{b^{2}}{4}+G\right)}\left[k(0, \theta) \mathrm{de}^{\frac{b \theta}{2}}+\mathrm{e}^{\frac{b \theta}{2}} \mathrm{~d} k(0, \theta)\right] \\
& =\frac{2}{b} \mathrm{e}^{t\left(A-\frac{b^{2}}{4}+G\right)}\left[\frac{b}{2} \int_{0}^{2 \pi} k(0, \theta) \mathrm{d} \theta+\int_{0}^{2 \pi} \mathrm{d} k(0, \theta)\right] \\
& =\mathrm{e}^{t\left(A-\frac{b^{2}}{4}+G\right)}\langle k(0, \theta), \mathbb{1}\rangle+k_{0}(2 \pi)-k_{0}(0) \\
& =\mathrm{e}^{t\left(A-\frac{b^{2}}{4}\right)} K(0) .
\end{aligned}
$$

The optimal aggregate capital stock grows at the rate $r=A-\frac{b^{2}}{4}$ from $t=0$. Combining Equation (35) and (64), the optimal control of the auxiliary problem could be formulated as $\hat{c}^{*}(t, \theta)=\eta \mathrm{e}^{\beta t} Z(0)$. Apply Lemma 1 again, optimal consumption is expressed as $c^{*}(t, \theta)=\eta \mathrm{e}^{-\frac{b \theta}{2}+\beta t} Z(0)$.

By substituting Equation (57) into the system (9), we have the following theorem.

Theorem 4 Under the same assumptions of Lemma 1 and 2, the optimal evolution of the capital distribution starting from $k_{0}$ is the solution of the following partial differential system

$$
\left\{\begin{array}{l}
\frac{\partial k(t, \theta)}{\partial t}=\frac{\partial^{2} k(t, \theta)}{\partial \theta^{2}}+b \frac{\partial k(t, \theta)}{\partial \theta}+A k(t, \theta)-\eta \mathrm{e}^{-\frac{b \theta}{2}+\beta t} Z(0), \\
k(0, \theta)=k_{0}(\theta), \theta \in \mathbb{T}, \\
k(t, 0)=k(t, 2 \pi), t \in[0,+\infty),
\end{array}\right.
$$

where $\eta, \beta$ and $Z(0)$ are given in Theorem 3.

Theorem 5 Under the hypotheses of Lemma 1 and 2, along the optimal trajectory, the detrended capital

$$
k_{D}(t, \theta):=k(t, \theta) \mathrm{e}^{-t\left(A-\frac{b^{2}}{4}\right)}
$$


converges uniformly, as a function of $\theta, \frac{\mathrm{e}^{-\frac{b \theta}{2}} Z(0)}{2 \pi}$. when $t$ tends to infinity, i.e.,

$$
\lim _{t \rightarrow \infty}\left(\sup _{\theta \in \mathbb{T}}\left|k_{D}(t, \theta)-\frac{\mathrm{e}^{-\frac{b \theta}{2}} Z(0)}{2 \pi}\right|\right)=0
$$

Proof For positive integer $n \in \mathbb{Z}$, we define the function $e_{n}: \mathbb{T} \rightarrow \mathbb{R}$ as

$$
e_{n}(\theta):=\left\{\begin{array}{l}
\frac{\cos (n \theta)}{\sqrt{\pi}}, n \geq 1, \\
\frac{\sin (-n \theta)}{\sqrt{\pi}}=\frac{\sin (n \theta)}{\sqrt{\pi}}, n \leq-1, \\
\frac{1}{\sqrt{2 \pi}}, n=0 .
\end{array}\right.
$$

It is obvious that $e_{n}(\theta) \in D(G)$ and $G e_{n}(\theta)=\frac{\mathrm{d}^{2} e_{n}(\theta)}{\mathrm{d} \theta^{2}}=-n^{2} e_{n}(\theta)$. For a fixed $\theta$, let $\omega(t, \theta):=\mathrm{e}^{t G} e_{n}(\theta)$, we have $\frac{\mathrm{d} \omega(t, \theta)}{\mathrm{d} t}=\mathrm{e}^{t G} G e_{n}(\theta)=-n^{2} \mathrm{e}^{t G} e_{n}(\theta)=-n^{2} \omega(t, \theta)$. So $\omega(t, \theta)=\mathrm{e}^{-n^{2} t} e_{n}(\theta)$, that is $\mathrm{e}^{t G} e_{n}(\theta)=\mathrm{e}^{-n^{2} t} e_{n}(\theta)$ which implies $\mathrm{e}^{t G} \mathbb{1}=\mathbb{1}$. Taking the scalar product of Equation (50) and $e_{n}$ for all $n$, we have

$$
\begin{aligned}
\left\langle z(t), e_{n}\right\rangle & =\left\langle\mathrm{e}^{t\left(A-\frac{b^{2}}{4}+G\right)} z_{0}, e_{n}\right\rangle-\int_{0}^{t}\left\langle\mathrm{e}^{(t-s)\left(A-\frac{b^{2}}{4}+G\right)} \eta\langle z(s), \mathbb{1}\rangle \mathbb{1}, e_{n}\right\rangle \mathrm{d} s \\
& =\left\langle z_{0}, \mathrm{e}^{t\left(A-\frac{b^{2}}{4}+G\right)} e_{n}\right\rangle-\int_{0}^{t} \eta\langle z(s), \mathbb{1}\rangle\left\langle\mathbb{1}, \mathrm{e}^{(t-s)\left(A-\frac{b^{2}}{4}+G\right)} e_{n}\right\rangle \mathrm{d} s .
\end{aligned}
$$

If $n \neq 0,\left\langle e_{n}, \mathbb{1}\right\rangle=0$ since it is the integral on $[0,2 \pi]$ of a constant times $\sin (n \theta)$ or $\cos (n \theta)$, combining with

$$
\mathrm{e}^{t G} \mathrm{e}^{\left(A-\frac{b^{2}}{4}\right) t} e_{n}(\theta)=\mathrm{e}^{\left(A-\frac{b^{2}}{4}-n^{2}\right) t} e_{n}(\theta),
$$

we have

$$
\left\langle z(t), e_{n}\right\rangle=\left\langle z_{0}, \mathrm{e}^{t\left(A-\frac{b^{2}}{4}-n^{2}\right)} e_{n}\right\rangle=\mathrm{e}^{t\left(A-\frac{b^{2}}{4}-n^{2}\right)}\left\langle z_{0}, e_{n}\right\rangle, n \neq 0 .
$$

If $n=0$, we have $\left\langle z(t), e_{0}\right\rangle=\frac{Z(0) \mathrm{e}^{t\left(A-\frac{b^{2}}{4}\right)}}{\sqrt{2 \pi}}$. Using Fourier series, $z(t)$ can be expressed as

$$
Z(t)(\theta)=\frac{Z(0) \mathrm{e}^{t\left(A-\frac{b^{2}}{4}\right)}}{2 \pi}+\sum_{n \in Z, n \neq 0} \mathrm{e}^{t\left(A-\frac{b^{2}}{4}-n^{2}\right)}\left\langle z_{0}, e_{n}\right\rangle e_{n}(\theta) .
$$


The detrended capital is written in the form

$$
\begin{aligned}
k_{D}(t, \theta) & =\mathrm{e}^{-t\left(A-\frac{b^{2}}{4}\right)} k(t, \theta)=\mathrm{e}^{-t\left(A-\frac{b^{2}}{4}\right)} Z(t)(\theta) u(\theta) \\
& =\mathrm{e}^{-t\left(A-\frac{b^{2}}{4}\right)} u(\theta)\left[\frac{\left.Z(0) \mathrm{e}^{t\left(A-\frac{b^{2}}{4}\right)}+\sum_{n \in Z, n \neq 0} \mathrm{e}^{t\left(A-\frac{b^{2}}{4}-n^{2}\right)}\left\langle z_{0}, e_{n}\right\rangle e_{n}(\theta)\right]}{2 \pi}\right] \\
& =\frac{Z(0) u(\theta)}{2 \pi}+\sum_{n \in Z, n \neq 0} \mathrm{e}^{-n^{2} t}\left\langle z_{0}, e_{n}\right\rangle e_{n}(\theta) u(\theta) .
\end{aligned}
$$

For $\forall \varepsilon>0$, using Cauchy-Schwartz inequality and $\left|z_{0}\right|_{L^{2}(\mathbb{T})}^{2}=\sum_{n \in \mathbb{Z}}\left|\left\langle z_{0}, e_{n}\right\rangle\right|^{2}$, we have

$$
\begin{aligned}
\sup _{\theta \in \mathbb{T}}\left|k_{D}(t)(\theta)-\frac{\mathrm{e}^{-\frac{b \theta}{2}} Z(0) \mid}{2 \pi}\right| & =\sup _{\theta \in \mathbb{T}}\left|\sum_{n \in Z, n \neq 0} \mathrm{e}^{-n^{2} t}\left\langle z_{0}, e_{n}\right\rangle e_{n}(\theta) u(\theta)\right| \\
& \leq \mathrm{e}^{-\varepsilon t}\left|\sum_{n \in Z, n \neq 0} \mathrm{e}^{t\left(-n^{2}+\varepsilon\right)}\right|\left\langle z_{0}, e_{n}\right\rangle\left|\sup _{\theta \in \mathbb{T}}\right| e_{n}(\theta) u(\theta)|| \\
& =\mathrm{e}^{-\varepsilon t}\left|\sum_{n \in Z, n \neq 0} \mathrm{e}^{t\left(-n^{2}+\varepsilon\right)}\right|\left\langle z_{0}, e_{n}\right\rangle|| \\
& \leq \mathrm{e}^{-\varepsilon t}\left(\sum_{n \in Z, n \neq 0} \mathrm{e}^{2 t\left(-n^{2}+\varepsilon\right)}\right)^{\frac{1}{2}}\left(\sum_{n \in Z, n \neq 0}\left|\left\langle z_{0}, e_{n}\right\rangle\right|^{2}\right)^{\frac{1}{2}} \\
& =\mathrm{e}^{-\varepsilon t}\left(\sum_{n \in Z, n \neq 0} \mathrm{e}^{t\left(-n^{2}+\varepsilon\right)}\right)^{\frac{1}{2}}\left|z_{0}\right|_{L^{2}} .
\end{aligned}
$$

Letting $S:=\sum_{n \in Z, n \neq 0} \mathrm{e}^{t\left(-n^{2}+\varepsilon\right)}<\infty$, By the inequality (75), we conclude that

$$
\sup _{\theta \in \mathbb{T}}\left|k_{D}(t)(\theta)-\frac{e^{-\frac{b \theta}{2}} Z(0)}{2 \pi}\right| \leq \mathrm{e}^{-\varepsilon t} S\left|z_{0}\right|_{L^{2}} \stackrel{t \rightarrow \infty}{\longrightarrow} 0 \text {. }
$$

Theorem 5 shows that the trade barriers do have an effect on the convergence of the spatial AK model. When $2 \sqrt{A-\frac{\rho}{1-\sigma}}<b<2 \sqrt{A}$, the detrended capital converges to $\frac{\mathrm{e}^{-\frac{b \theta}{2}} Z(0)}{2 \pi}$. When $b=0$, the detrended capital converges to $\frac{K(0)}{2 \pi}$. When trade costs exceed the reasonable range, the detrended capital may not be convergent. The next section provides a complementary simulation verification.

\section{Numerical Illustration}

To illustrate the dynamics of the spatial AK model with trade costs, we provide several numerical examples by using the explicit optimal dynamics of the detrended capital $k_{D}(t, \theta)$ in the form of Fourier series. In the following exam- 
ples, there are four key parameters in our modeling: $A, b, \rho$ and $\sigma$. we choose $A=0.36$ as a reasonable ratio output to capital and fix $\rho=0.06, \sigma=0.8$. We choose two different trade barrier coefficients $b$ on the condition that the set $A, b, \rho, \sigma$ satisfies hypothesis (33) and analyze the impacts of trade costs on capital accumulation with different initial distributions of capital.

Example 1 For a fixed trade cost coefficient, set $b=\frac{1}{2}$ and compare the influence of trade cost coefficient on capital accumulation with different endowments of capital. We consider three different initial capital distribution $k_{0}(\theta)$, when $k_{0}(\theta)=|\theta \cos \theta|$, the detrended capital behaves as in Figure 1, when $k_{0}(\theta)=\theta^{2}$, the trend of the physical capital is shown in Figure 2, see Figure 3 when $k_{0}(\theta)=|\cos \theta|$.

From Figures 1-3, it can be seen that the distrended capital $k_{D}(t, \theta)$ under the different initial distributions have the same trend. Next, we change the trade cost coefficient $b$.

Example 2 Might as well take $b=1$ and consider the three different initial capital distributions $k_{0}(\theta)$ as the same in Example 1, the dynamic of the corresponding detrended capital is illustrated in Figures 4-6.

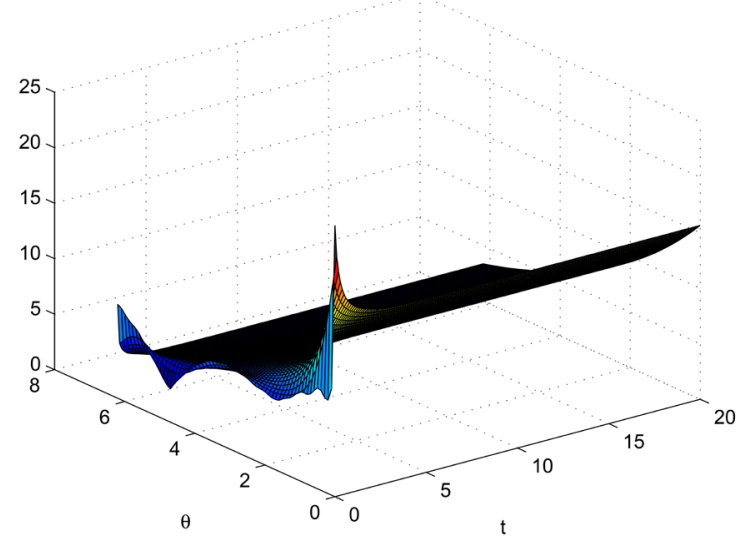

Figure 1. $k_{D}(t, \theta)$, when $k_{0}(\theta)=|\theta \cos \theta|$.

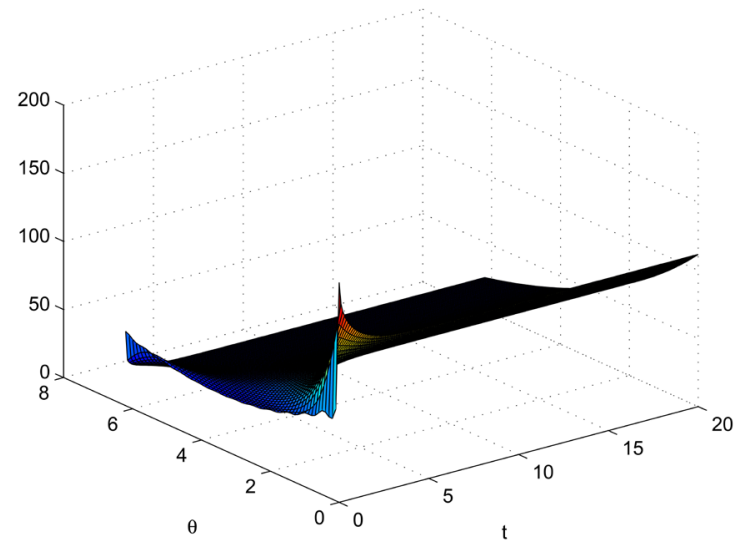

Figure 2. $k_{D}(t, \theta)$, when $k_{0}(\theta)=\theta^{2}$. 


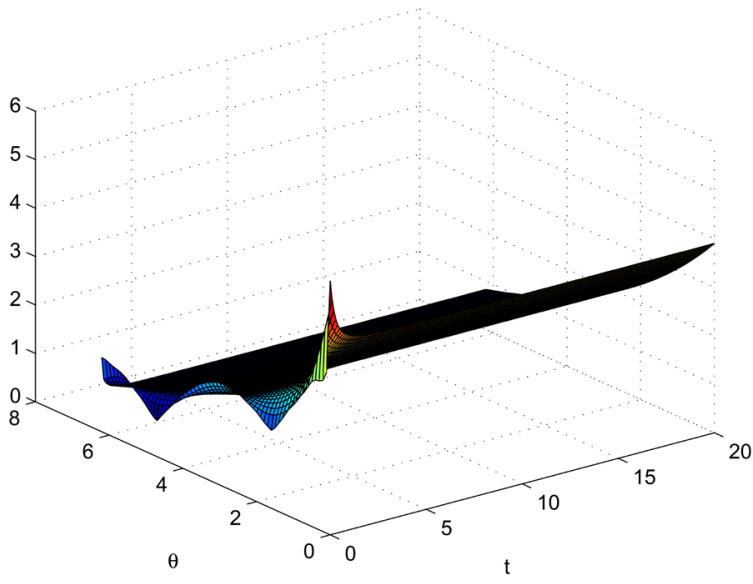

Figure 3. $k_{D}(t, \theta)$, when $k_{0}(\theta)=|\cos \theta|$.

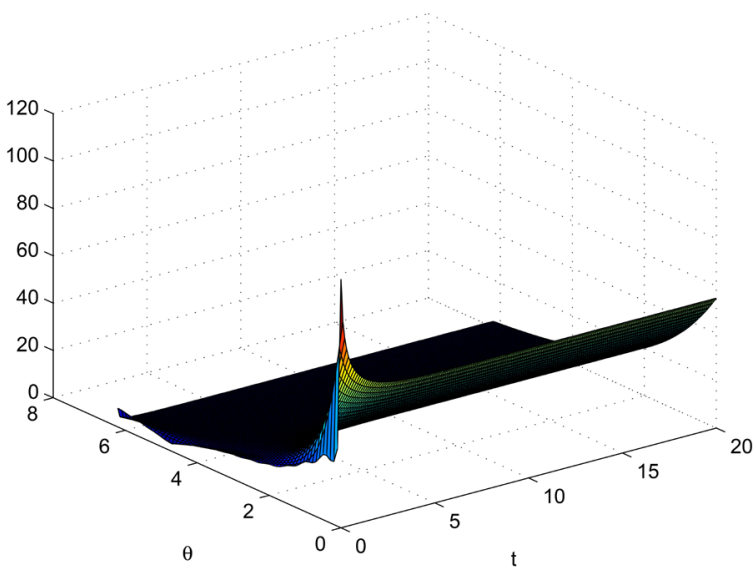

Figure 4. $k_{D}(t, \theta)$, when $k_{0}(\theta)=|\theta \cos \theta|$.

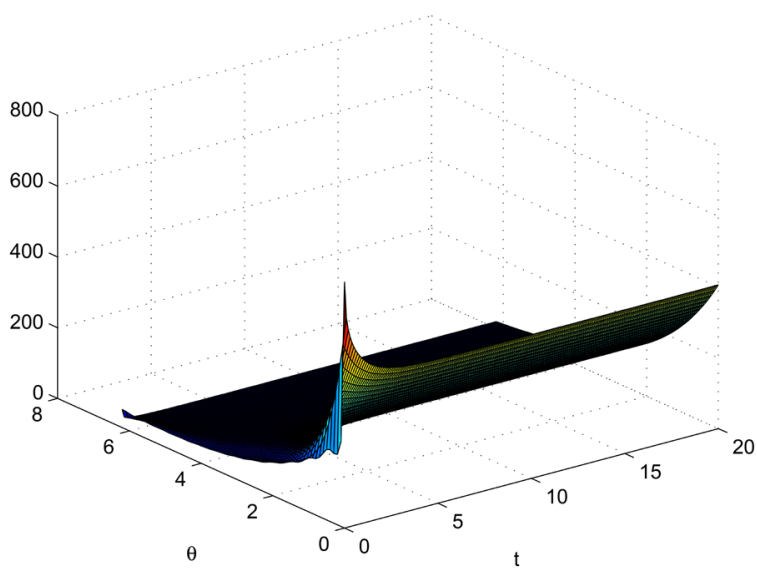

Figure 5. $k_{D}(t, \theta)$, when $k_{0}(\theta)=\theta^{2}$.

Moreover, we analyze the evolution of the detrended capital as the cost coefficient changes. With the same initial distribution, compare Figures 1-6, we find that the bigger the cost coefficient $b$ is, the slower the convergence rate of $k_{D}(t, \theta)$ is, trade costs have an effect on the dynamics of capital accumulation. 


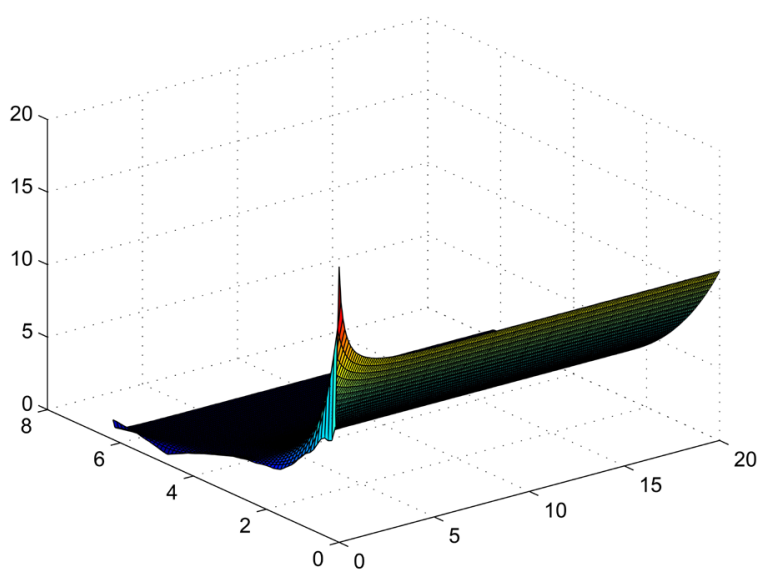

Figure 6. $k_{D}(t, \theta)$, when $k_{0}(\theta)=|\cos \theta|$.

\section{Conclusion}

In this work, we investigate the role of trade costs in the evolution of a spatial growth model. To this extent, we formulate an optimal control problem with the dynamics of physical capital growth as constraints. By employing the dynamic programming method and semigroup theory, we obtain the optimal consumption strategy and capital growth trajectory with trade costs. We also find that when the trade cost keeps within a reasonable range, the spatiotemporal dynamics reduce the inequalities in capital endowments and lead to the convergence of capital over space in the long run. Finally, we perform numerical simulations to support the analytical results and illustrate the spatiotemporal dynamics generated by the model. In addition, if trade cost is a nonlinear function of $k(t, \theta)$, we will face a more complicate and challenging problem which needs further study.

\section{Acknowledgements}

The authors are very grateful to the reviewers for their helpful and valuable comments, which have led to a meaningful improvement of the paper.

\section{Conflicts of Interest}

The authors declare no conflicts of interest regarding the publication of this paper.

\section{References}

[1] Bywaters, D. and Mlodkowski, P. (2012) The Role of Transactions Costs in Economic Growth. International Journal of Economic Policy Studies, 7, 53-66. https://doi.org/10.1007/BF03405737

[2] Fratianni, M. and Marchionne, F. (2012) Trade Costs and Economic Development. Economic Geography, 88, 137-163. https://doi.org/10.1111/j.1944-8287.2012.01145.x

[3] Chandra, P. (2016) Impact of Temporary Trade Barriers: Evidence from China. 
China Economic Review, 38, 24-48. https://doi.org/10.1016/j.chieco.2015.11.002

[4] Storeygard, A. (2016) Farther on Down the Road: Transport Costs, Trade and Urban Growth in Sub-Saharan Africa. The Review of Economic Studies, 83, 1263 1295. https://doi.org/10.1093/restud/rdw020

[5] Lai, C., Fan, H. and Qi, H. (2020) Global Gains from Reduction in Trade Costs. Economic Theory, 70, 313-345. https://doi.org/10.1007/s00199-019-01211-w

[6] Brancaccio, G., Kalouptsidi, M. and Papageorgiou, T. (2020) Geography, Transportation, and Endogenous Trade Costs. Econometrica, 88, 657-691.

https://doi.org/10.3982/ECTA15455

[7] Brito, P. (2004) The Dynamics of Growth and Distribution in a Spatially Heterogeneous World. Working Papers Department of Economics. https://core.ac.uk/download/pdf/7027939.pdf

[8] Boucekkine, R., Camacho, C. and Zou, B. (2009) Bridging the Gap between Growth Theory and the New Economic Geography: The Spatial Ramsey Model. Macroeconomic Dynamics, 13, 20-45. https://doi.org/10.1017/S1365100508070442

[9] Camacho, C., Zou, B. and Briani, M. (2008) On the Dynamics of Capital Accumulation across Space. European Journal of Operational Research, 186, 451-465. https://doi.org/10.1016/j.ejor.2007.02.031

[10] Boucekkine, R., Camacho, C. and Fabbri, G. (2013) Spatial Dynamics and Convergence: The Spatial AK Model. Journal of Economic Theory, 148, 2719-2736. https://doi.org/10.1016/j.jet.2013.09.013

[11] Boucekkine, R., Fabbri, G., Federico, S. and Gozzi, F. (2019) Growth and Agglomeration in the Heterogeneous Space: A Generalized AK Approach. Journal of Economic Geography, 19, 1287-1318. https://doi.org/10.1093/jeg/lby041

[12] Ballestra, L. (2016) The Spatial AK Model and the Pontryagin Maximum Principle. Journal of Mathematical Economics, 67, 87-94. https://doi.org/10.1016/j.jmateco.2016.09.012

[13] Juchem Neto, J. and Claeyssen, J. (2015) Capital-Induced Labor Migration in a Spatial Solow Model. Journal of Economics, 115, 25-47.

https://doi.org/10.1007/s00712-014-0404-6

[14] Juchem Neto, J., Claeyssen, J. and Pôrto Júnior, S. (2018) Economic Agglomerations and Spatio-Temporal Cycles in a Spatial Growth Model with Capital Transport Cost. Physica A: Statistical Mechanics and Its Applications, 494, 76-86. https://doi.org/10.1016/j.physa.2017.12.036

[15] Xepapadeas, A. and Yannacopoulos, A. (2016) Spatial Growth with Exogenous Saving Rates. Journal of Mathematical Economics, 67, 125-137. https://doi.org/10.1016/j.jmateco.2016.09.010

[16] Fabbri, G. (2016) Geographical Structure and Convergence: A Note on Geometry in Spatial Growth Models. Journal of Economic Theory, 162, 114-136. https://doi.org/10.1016/j.jet.2015.12.004

[17] Desmet, K. and Rossi-Hansberg, E. (2010) On Spatial Dynamics. Journal of Regional Science, 50, 43-63. https://doi.org/10.1111/j.1467-9787.2009.00648.x

[18] Bensoussan, A., Prato, G., Delfour, M. and Mitter, S. (2007) Representation and Control of Infinite Dimensional Systems. Birkhäuser, Boston. https://doi.org/10.1007/978-0-8176-4581-6 